\title{
Un meta-análisis de los efectos de las TIC sobre el nivel de empleo y los roles laborales: comentarios y direcciones futuras
}

\author{
Guillermo Alberto, Tricoci \\ Universidad de Buenos Aires, Argentina
}

Recibido: 31 de marzo de 2014

Aceptado: 17 de octubre de 2014

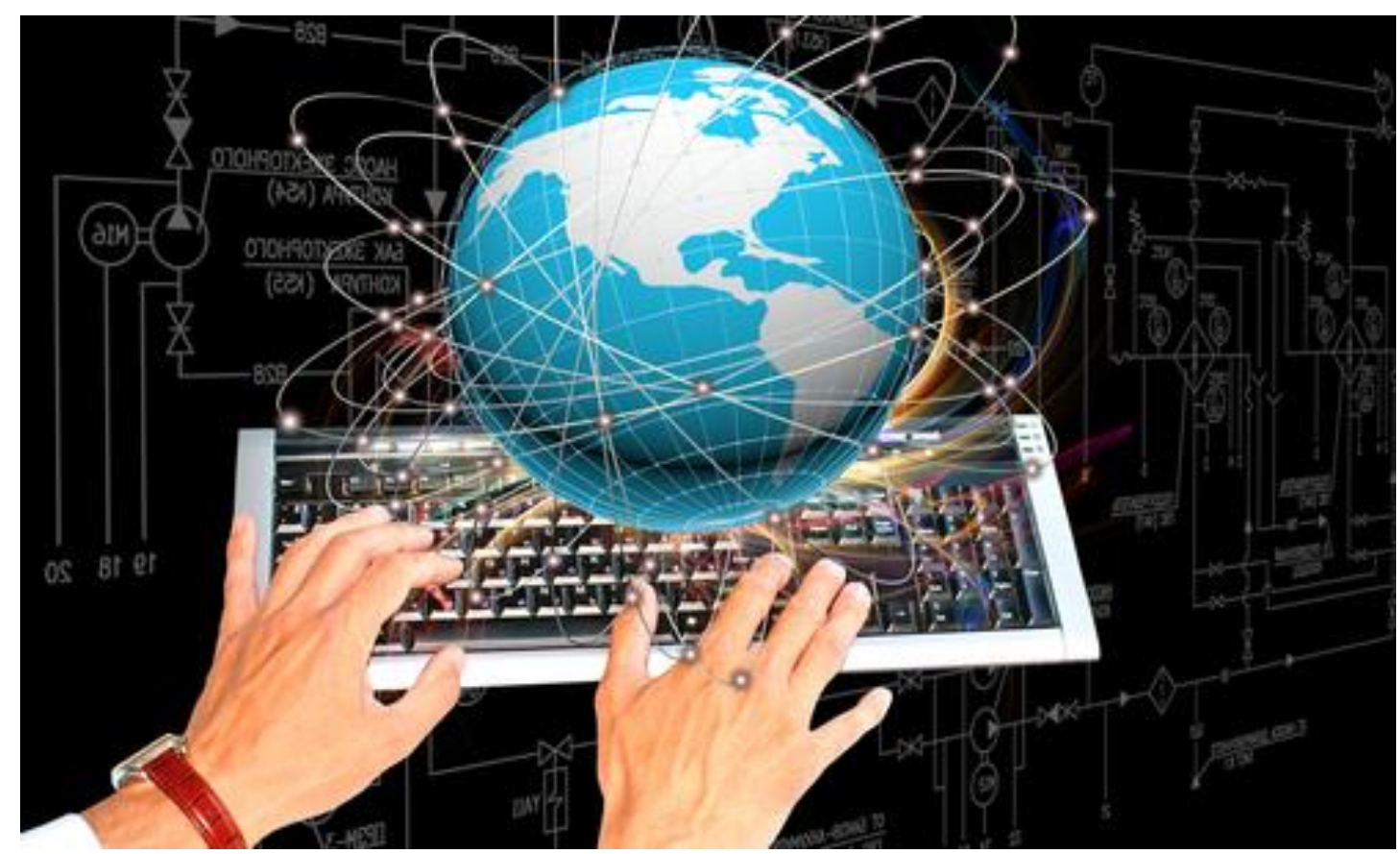

Imágenes utilizadas bajo licencia de uso por Shutterstock.com

\begin{abstract}
Similarly to what happened with the steam engine, the electricity or the combustion engine, the new general-purpose technologies and ICTs have introduced many fundamental changes to our life and the processes we deal with daily. They have provided many benefits but have also produced unwanted collateral effects that should be considered. The first effect, at the beginning there was a new phenomenon known as the digital divide. Later on the uncertainty around the true scope of computing increased and evidence of a decrease in the capability of creating new jobs and stagnant or falling wages became clearer. All these, amongst other things, are considered part of the ICT revolution. The paradigm of continuous improvement to innovation and productivity begins to show the effects it produces in the disappearance and transformation of business roles. Many activities have disappeared and much of the business processes have received a very high degree of automation. These changes can be seen in the most varied fields.
\end{abstract}

KEY WORDS: ICT, digital divide, employment, business processes 


\section{RESUMEN}

Como lo fueron anteriormente los impactos de la máquina a vapor, la electricidad y el motor a combustión, las nuevas tecnologías de propósito general han producido cambios trascendentales; en esa línea las TIC están cambiando muchos de los procesos y actividades de nuestras vidas. Debido a ellas, gozamos de muchos beneficios, pero también han producido efectos no deseados que deben ser atendidos especialmente. En un principio fue la aparición de un nuevo fenómeno conocido como la brecha digital. Luego se agregaron incertidumbres sobre los verdaderos alcances de las computadoras, y en los últimos tiempos aparecen indicios de limitaciones en la creación de empleos y el estancamiento o caída en los salarios, asociados, entre otras variables, a la revolución de las TIC. El paradigma de la mejora continua hacia la innovación y aumento de la productividad, comienza a mostrar los impactos que produce en la desaparición y transformación de roles laborales. Muchas actividades han desaparecido y gran parte de los procesos de negocio han recibido un grado de automatización muy elevado. Estos cambios podemos verlos en los más variados ámbitos.

Palabras claves: TIC, brecha digital, empleo, procesos de negocio

\section{Introducción}

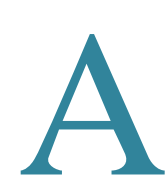

1 igual que los impactos producidos por la máquina a vapor, la
electricidad y el motor a combustión, las tecnologías de la información y la comunicación (en adelante TIC, y refiere a los elementos equipo, programas, comunicaciones $\mathrm{y}$ sus servicios asociados) cambian muchos de los procesos y actividades de nuestras vidas. Debido a ellas, gozamos de innumerables beneficios, pero también han aparecido efectos no deseados que deben ser atendidos especialmente.
En un principio surgió un nuevo fenómeno conocido como la brecha digital. Luego se agregaron incertidumbres y miedos sobre los verdaderos alcances de las computadoras en los trabajos humanos, y últimamente aparecen indicios de limitaciones en la creación de empleos y del estancamiento o caída en los salarios, asociados, entre otras variables, a la revolución de las TIC. El paradigma de la mejora continua hacia la innovación y el aumento sistemático de la productividad, comienza a mostrar los impactos que se traducen en la desaparición y transformación de roles laborales. Muchas actividades han desaparecido y gran parte de los procesos de negocio han recibido un grado de automatización muy elevado. Estos cambios podemos verlos en los más variados ámbitos.

La automatización de los distintos procesos relacionados con los viajes en avión es un ejemplo de lo comentado previamente. Hoy podemos comprar pasajes aéreos por un sitio web, realizar su pago con tarjeta de crédito, administrar el billete / pasaje electrónico (su cambio si fuese necesario, la asignación de asientos, menú de comidas especiales), realizar el registro en los vuelos, auto-facturar el equipaje (self-drop off), y podemos entrar y salir de un país sin intervención humana mediante el uso de los pasaportes electrónicos. En lo referido a los servicios turísticos agregados, como reserva de hoteles, alquiler de auto, etc., todos los procesos se realizan con mínima o nula intervención humana. Este nuevo esquema presenta para las empresas, organizaciones prestadoras de los servicios referidos, una importantísima reducción y automaticidad de sus procesos internos 
(back office) los cuales se han modificado fuertemente, lo que permite un increíble aumento de la productividad, creación de nuevos servicios y mejora de los existentes.

Similares comentarios podemos hacer respecto del proceso de ir al cine: buscamos en algún sitio de la web la tienda de venta de entradas, seleccionamos una función y un asiento disponible para ver la película desde una buena ubicación y colocamos nuestra tarjeta de crédito para el pago de las entradas. Una vez completada la operación, recibimos un código QR, para nuestro celular, que nos permitirá entrar directamente a la sala. Podemos seguir con ejemplos de distintos procesos del área financiera, o de las áreas de manufactura (ej., control de procesos, robot), de logística, la relación con los organismos gubernamentales, la re-conversión y/o casi extinción de industrias, como la fotográfica física, la discográfica, entre muchos otros casos y abarcan muchos sectores de la economía de un país.

Los cambios provocaron, en su gran mayoría, la desaparición de muchos puestos de trabajo manuales que se requerían con anterioridad $y$ que hoy han sido reemplazados por las nuevas tecnologías, con la creación de nuevos roles, pero infinitamente menores en su cantidad nominal respecto de los reemplazados. Como contrapartida, los clientes y toda la sociedad reciben mejores servicios y a costos más bajos (en algunos casos cercanos a cero).

Existen indicios de que estos aumentos de la productividad no conllevan mejores ingresos de los trabajadores medios y de que, a la vez, hay aumentos importantes de los niveles de desempleo y caída en las tasas de la generación de puestos de trabajo. Esta alarma se pudo verificar en la salida del proceso recesivo del 2008-2009 en EEUU, y en especial con referencia a la recuperación del nivel de empleo.

La desaparición de roles laborales, la modificación de otros, pero básicamente la automatización de procesos nos recuerda las palabras de J. M. Keynes (1930): "Estamos siendo afectados por una nueva enfermedad de la que algunos lectores todavía pueden no haber escuchado el nombre, pero de la cual escucharemos mucho en los próximos años, a saber: el desempleo tecnológico. Esto significa que el desempleo, debido a nuestros descubrimiento de medios de economizar la utilización de mano de obra está dejando atrás el ritmo por el cual podemos encontrar nuevos usos para el trabajo."

El objetivo del presente artículo es analizar la evolución de uso de las TIC en referencia al impacto que producen en los roles laborales a partir de visiones de distintos autores que aportan miradas desde la tecnología y la economía.

\section{Las primeras dificultades.}

La masificación del uso de las TIC ha traído aparejada la aparición de un nuevo concepto conocido como la brecha digital. En Tricoci (2011) decía que "La era digital y el impacto mundial de las TIC pueden tener aspectos negativos. En la medida que el acceso a la tecnología tenga restricciones, aparece el peligro de una nueva y peligrosa forma de exclusión social. ¿Es posible un futuro con personas que desconozcan el lenguaje digital?"

Van Dijk y Hacker (2003) señalan cuatro problemas relacionados con el acceso:

- Acceso mental: se caracteriza por la 
falta de experiencia digital elemental, causada por la ausencia de interés o de atracción de la nueva tecnología.

- Acceso material: se describe como la imposibilidad de disponer de computadoras y conexión de red. Es la "no-posesión" de los medios de procesamiento y su conexión.

- Acceso de habilidades: está representado por la falta de capacidades debida a la ausencia de educación o educación inadecuada, falencia en el apoyo social, o conocimiento insuficiente del usuario de la tecnología.

- Acceso para el uso: puede no tener problemas de acceso mental, material o de habilidades, pero no tiene oportunidades de uso significativo y por lo tanto su conocimiento se vuelve obsoleto o es insuficiente para saltar la barrera de lo aplicativo.

¿Cómo impacta en el mercado laboral la existencia de la brecha digital?

El resultado es un mercado dual con, por lo menos, dos segmentos:

- Segmentos de los conectados: en este segmento se encuentra la oferta laboral que tiene acceso a las nuevas tecnologías, que tiene habilidades de uso $y$ que puede extraer sus potencialidades. Son quienes conocen y manejan el alfabeto digital. Es un segmento de alta especialización tecnológica, posible escasez de oferta (según localizaciones y contextos), mayor demanda y mejores salarios.

- Segmento de los desconectados: el mismo comprende a los que tienen limitado el acceso a las nuevas tecnologías y que no pueden adquirir las competencias y habilidades necesarias para su uso. Son, en cierta forma, los analfabetos digitales. En oposición al segmento anterior, encontramos una baja calificación laboral, posible precariedad de empleo y bajos salarios.

El acceso de los desconectados al segmento de los conectados tiene un costo de entrada cada vez mayor. Los desconectados, por sí solos y sin ayuda, ven cada vez más lejos la posibilidad de realizar el catch up.

La reducción o eliminación de las brechas deben ser objetivos de políticas de estado, lo que implica acciones en múltiples frentes; las soluciones de los mercados son ineficientes como única alternativa a la resolución de este problema. Se ha avanzado en la instalación de este tema, y forma parte de la agenda pública de la mayoría de los países, en los cuales los gobiernos deben intervenir para reducir y eliminar esta brecha.

¿Cuáles son los límites de las computadoras, hasta dónde llegarán?

Uno de los hitos más relevantes de este avance de la tecnología de la información podemos encontrarlo en la famosa Ley de Moore. Moore enunciaba que la cantidad de transistores integrados al mínimo costo, se duplicaba cada 12 meses. Posteriormente se comprobó que el período señalado por la ley no era de 12 meses sino de 18 meses. Convivimos con una tecnología que puede duplicar su potencia y/o de hacer caer sus costos cada año y medio. Similares conceptos podemos aplicar a los demás componentes de un sistema de cómputos y también al desarrollo de software. Estamos a casi 50 años de ese momento, a medio siglo 
de la expresión de una ley que enunciaba un concepto exponencial del crecimiento.

Brynjolfsson y Mc Afee (2011) plantean en su trabajo la idea de que el crecimiento exponencial hace perder la perspectiva de lo posible y de cuán lejos o cerca estamos de alcanzar nuevos logros. Lo que parece lejano o imposible de lograr, transcurrido cierto tiempo se acerca con una velocidad no prevista. Lo que era imposible o ficción pasa a estar a la vuelta de la esquina. Los avances de las computadoras han sido increíbles en los últimos tiempos, pero ¿qué cosas pueden hacer las computadoras?, ¿cuáles son los límites?

En una simplificación importante, podemos caracterizar a la automatización de procesos o tareas en la medida en que se puedan tomar los datos mediante alguna forma de representación certera y precisa (scanners o sensores), y que la función de procesamiento pueda ser expresada con algoritmos o transformada en reglas, ya sea en forma deductiva o inductiva. La forma deductiva se logra estudiando y descubriendo la secuencia lógica del proceso. Es deductiva porque se llega en forma descendente a obtener los resultados posibles. Un ejemplo de un proceso de negocios puede ser la aprobación de un pedido de venta de un cliente, donde la secuencia de pasos del proceso finaliza con el pedido aprobado o rechazado.

En el caso de la forma inductiva, existe un desconocimiento del resultado final y se define, por ejemplo, mediante el reconocimiento de patrones o estructuras (pattern recognition). Puede referirse a ecuaciones de regresión, redes neuronales o modelos estadísticos para la obtención de parámetros de comportamiento. Las ecuaciones con los parámetros estimados permiten ser reutilizadas para nuevos casos.
Como ejemplo pensemos en la determinación de patrones de uso de las tarjetas de crédito para avisar sobre los potenciales fraudes.

Los ejemplos de automatizaciones vistos en la introducción (viajes en avión, salida al cine, procesos bancarios, etc.), cumplen las condiciones anteriores, mayormente por la aplicación de reglas deductivas: sensores, lectores inteligentes, almacenamiento de datos previos en dispositivos de lectura posterior, dispositivos inteligentes, la registración de los hechos por el causante (Cuando compramos un pasaje o una entrada de cine realizamos nosotros la captura de datos que antes realizaba un empleado de la empresa.), etc. Estos procesos automatizados tienen incluido conocimiento que fue explicitado y convertido en software.

Existe otro tipo de procesos de más difícil automatización donde intervienen otros elementos de mayor complejidad y sobre los cuales hay aún barreras para su modelaje, o su coste es muy alto.

\section{¿Cualquier conocimiento puede ser convertido en software?}

En el punto anterior nos referimos a procesos que incluían conocimiento. ¿Qué tipo de conocimiento es susceptible de ser expresado con algoritmos? Michael Polanyi (1962) realizó una clasificación del conocimiento en tácito y explícito. Explicaba que el conocimiento tácito es el que la persona tiene incorporado sin tenerlo permanentemente accesible a la conciencia, pero del cual hace uso cuando lo requiere. Es utilizado como instrumento para manipular el fenómeno que se analiza. Es el estado natural de conocimiento y está constituido por todo lo que no puede ser 
expresado, articulado o formalizado, como las habilidades personales, los procesos habituales y las costumbres. Podemos hacer más de lo que decimos que hacemos, son esas cosas que resolvemos o decidimos sin poder explicar claramente su forma.

El conocimiento explícito es aquel conocimiento objetivo y racional que puede ser expresado en palabras, oraciones, números o fórmulas. Es el conocimiento que se puede expresar con algoritmos. Este es el tipo de conocimiento que fue el más transmitido desde siempre, pero en la era digital se aceleró su distribución, su acceso y disposición crecieron en forma exponencial. Definido explícitamente, el conocimiento es susceptible de ser aplicado por medios automáticos. Es patrimonio de los seres humanos explotar al máximo nuestra capacidad de manejar nuestro conocimiento tácito.

\section{¿Qué más podemos decir sobre las limitaciones a la automatización?}

Levy y Murnane (2005) plantearon limitaciones a las automatizaciones, e identificaron varios casos donde las computadoras tienen aún dificultades.

\section{- Limitaciones para representar datos de la realidad.}

Cuando realizamos una consulta, nos pueden pedir completar un cuestionario. El mismo puede ser respondido ante un computador, pero es necesario el interrogatorio del médico y la revisión para detectar otras señales (miradas, tono de voz, reflejos, etc.) que puedan completar, confirmar los datos de la encuesta inicial. Es el conocimiento tácito del médico aplicado sobre un protocolo o proceso determinado.

Otro ejemplo es el caso de los mecánicos especialistas en la detección $\mathrm{y}$ arreglos de fallas que no son detectadas por el software de escaneo de automóviles $\mathrm{u}$ otro tipo de productos que pretendemos reparar y que tiene alto contenido de componentes electrónicos. Estos especialistas pueden completar situaciones no contempladas en el software o que no fueron incluidas.

- Alto nivel de interacción humana y con situaciones con distintos grados de ambigüedad.

Si bien es posible la reducción de la ambigüedad, existen límites, hay situaciones en las cuales estas reducciones no son totalmente posibles/ por ejemplo, el personal de limpieza que va a un lugar nuevo, debe reconocer el lugar y luego resolver cuáles son los mejores pasos a seguir. Actúa el conocimiento tácito acumulado.

Como podemos ver, existen roles o tareas asociadas a la necesidad de determinación de patrones de comportamiento o de compleja comunicación, que no sólo pueden darse en trabajos de alta especialización, sino también en los de baja especialización.

\section{¿Cómo se afectan los roles laborales?}

Levy y Murnane (2005) presentan un trabajo sobre lo sucedido en EE.UU. entre 1969 y 1999 en los roles laborales. Las caídas más relevantes, como era de esperar, se dan en los trabajadores operarios manuales y los empleados de soporte administrativo.

$\mathrm{Si}$ analizamos las actividades de una organización como si fuera una pirámide con tres partes, como se muestra en la Figura 1, en la base de las misma se realizan los procesos rutinarios, compuestos por la 
toma de decisiones programables, y que son las más proclives a sistematizar y donde el trabajo humano es más fácilmente reemplazable por las computadoras. El nivel operativo es el que sufrió más fuertemente la automatización. Estas tareas son básicamente de los siguientes tipos:

- Rutinas cognitivas (routine cognitive tasks): requieren conocimiento que pueden ser descrito con reglas deductivas 0 inductivas. Estas actividades son las primeras en ser potencialmente automatizadas.

- Rutinas manuales (routine manual tasks): actividades manuales o físicas que pueden describirse con reglas deductivas o inductivas. En la medida que puedan ser descritas como una serie de movimientos repetitivos, son posibles de ser algoritmizadas con más facilidad

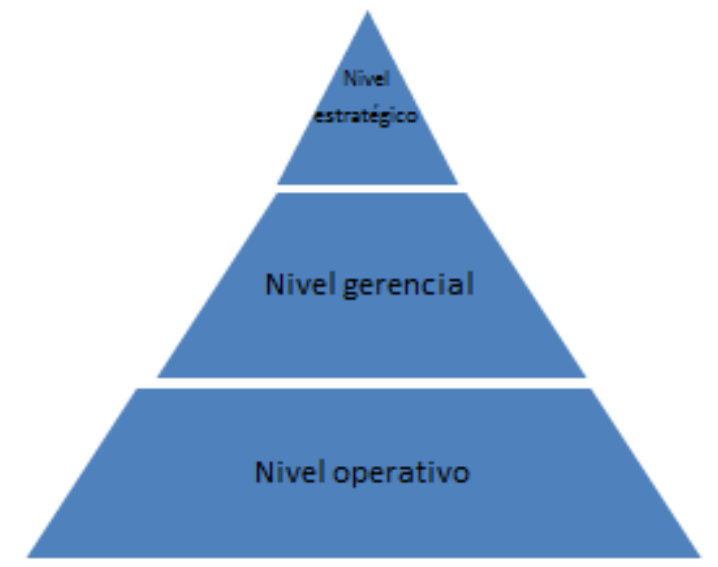

Figura 1. Actividades de una organización

La aparición de los sistemas de gestión del conocimiento e inteligencia de negocios apunta a los niveles medios y superiores, lo que elimina, en una primera instancia, las tareas rutinarias de las decisiones semiestructuradas y no estructuradas, y luego ayudan a tomar esas decisiones a partir de la evaluación de múltiples escenarios. Los sistemas expertos son también una alternativa para reemplazar las decisiones en varios de los niveles de la organización. Los niveles gerenciales intermedios empiezan a estar "acosados" por las máquinas en sus actividades semi-estructuradas.

\section{Las TIC, la macroeconomía y el empleo}

Los impactos de las TIC en la productividad variaron desde la década de los 80 cuando en los comienzos de la revolución de las computadoras, Solow (1987) dijo que se veían "computadoras en todas parte menos en la estadísticas de productividad" hasta nuestros días cuando su influencia es indiscutible. Desde la recesión económica del 2008-2009, en EE.UU han comenzado a aparecer una multiplicidad de informes sobre la recuperación económica y el impacto de las TIC en dicha salida.

En septiembre de 2011, el Wall Street Jornal publicó un artículo con el título It's Man vs. Machine and Man Is Losing. En el artículo de Kathleen Madigan se muestra que si se toman los datos del 2do trimestre 2009 y 2 do trimestre 2011 se puede ver que, desde el fin de la recesión, las empresas han invertido en bienes de capital (incluye TIC) un $26 \%$ más que en comienzo del período, mientras que los pagos de salarios se mantuvieron casi constantes. Con respecto a lo sucedido con los salarios, es más o menos coherente con lo referido a la creación de nuevos empleos en el período expuesto.

Estos datos parecen mostrar el optimismo de las firmas respecto de la recuperación, por el cual aumentaron sus gastos de capital, pero ese crecimiento de la producción no fue acompañado con mejoras en el empleo. Este cambio en la forma como se producen las salidas de la recesión, 
presenta un cambio respecto de las lecciones aprendidas. Arthur Okun en 1962 dio a conocer un trabajo que luego se popularizó como la Ley de Okun. Presentaba una regla simplificada respecto de qué sucedía con las variaciones entre la tasa de crecimiento del producto bruto de la economía y la tasa de desempleo. Preveía que una variación de 3 puntos del producto (en la tendencia de largo plazo) se correspondía con una variación de desempleo del $1 \%$ en el mismo sentido. Dicha formulación operaba tanto para las caídas como para las recuperaciones.

Robert Gordon (2010) publica una revisión de la Ley de Okun, donde confirma que la misma varía y que, si bien tuvo aplicabilidad hasta los 90, la situación ha cambiado últimamente. Una de las explicaciones de dicho cambio refiere a la Revolución de las TIC que ha permitido a las empresas una mayor flexibilidad y una explosión de productividad que les permitió reducir costos laborales, aún en los períodos de recuperación económica. Esto permite suponer que las recuperaciones del empleo serán cada vez más lentas.

McAfee y Brynjolfsson (2008) muestran que el stock de IT en el 2005 era el triple que en 1975. La inversión en TI por trabajador creció de US\$ 3500 en el año 1994 a U\$S 8000 en el año 2005: otro indicador del avance de las máquinas sobre el trabajo humano. El creciente uso de las TIC comienza a afectar los niveles de empleo y agrega un desafío extra al trabajo humano, del cual se requieren crecientes niveles de calificación. Se afectan los salarios también por la competencia con las máquinas.

\section{Conclusiones}

Cumplimos más de medio siglo con las computadoras, y gozamos como consumidores y ciudadanos de los tremendos cambios y beneficios que nos han proporcionado a la mayoría de la población. El uso de las TIC ha producido la desaparición de algunos roles laborales y la disminución de otros, la gran mayoría de ellos localizados en la parte inferior y medía de la teórica pirámide organizacional y se han creado nuevos roles, la gran mayoría asociados a la nueva industria TIC, pero en menor magnitud. Estos procesos identificados como shumpeterianos tienen en muchos casos una gran desproporción entre el trabajo destruido y el creado.

Las TIC están contribuyendo en el proceso de innovación y mejora de la competitividad de las firmas y esta tendencia se potenciará en el futuro. Los gerentes de las organizaciones deben tener presente que existe la creencia de que aún las firmas no aprovechan toda la potencialidad de las TIC en la mejora de sus procesos y que la brechas de mejoras son aún importantes, lo que implica que la modificaciones y reemplazo de trabajo humano continuará e incluso se incrementará en el futuro.

Los gobiernos y las sociedades deben pensar y coordinar políticas públicas para atender esta situación donde la desaparición de roles laborales será mayor que la creación de nuevas alternativas Las respuestas a esta nueva situación parecen estar del lado de producir una alianza virtuosa con las máquinas, en vez de luchar contra ellas como lo hicieron los luddistas (seguidores de Ned Ludd, quienes destruían telares mecanizados), a principios del siglo XIX en Inglaterra.

Dicha alianza debe tener como fundamentos la mejora continua de la educación, sin descuidar el mejoramiento de las destrezas básicas y las interacciones personales (fuertemente impactadas por la 
tecnología), los incentivos a la innovación, los emprendimientos y políticas públicas que incluyan en sus diagnósticos y programas esta nueva realidad, que ha llegado no sólo para quedarse, sino para profundizarse en el futuro.

\section{Agradecimiento}

Agradezco el intercambio de ideas, nuevos aportes y revisión de este trabajo realizado por la Lic. Noelia Massone.

\section{Referencias}

Autor, D., Levy, F. y Murnane, R.J. (2003). The skill content of recent technological change: An empirical exploration. The Quarterly Journal of Economics, 118(4), 1279-1333.

Brynjolfsson, E. y Mc Afee, A. (2011). Race against the machine. How the Digital Revolution is Accelerating Innovation, Driving Productivity, and Irreversibly Transforming Employment and the Economy. Lexington, Mass.: Digital Frontier Press.

Gordon, R. J. (2010, mayo) Okun's law and productivity innovations. American Economic Review: Papers \& Proceedings, 11-15. Recuperado de

http://www.aeaweb.org/articles.php?doi=10.1257/a er.100.2.11.

Keynes, J. (1930). Economic possibilities for our grandchildren. Essays in Persuasion (pp. 358-373). New York: W.W.Norton y Co.

Levy, F. y Murnane, R. J. (2005). How computerized work and globalization shape human skill demands. IPC Working PaperSeries IPC-05-006. Cambridge, MA: Massachusetts Institute of Technology.

Madigan, K. (2011, 28 de septiembre). It's man vs. machine and man is losing. The Wall Street Journal. Recuperado de http://blogs.wsj.com/economics/2011/09/28/itsman-vs-machine-and-man-is-losing/.

McAfee, A. y Brynjolfsson, E. (2008, July). Investing in the IT that makes a competitive difference. Harward Business Review. Recuperado de http://hbr.org/2008/07/investing-in-the-it-thatmakes-a-competitive-difference/ar/1.

Moore, G. E. (1998). Cramming more components onto integrated circuits. Proceedings of the 1998 IEEE, 86(1), 82-85.

Okun, Arthur M. "Potential GNP: Its Measurement and Significance". Proceedings of the Business and Economic Statistics Section of the American Statistical Association. Alexandria, VA: American Statistical Association, 1962, pp. 89-104.

Polanyi, Michal (1962). Personal Knowlegde: Toward a Post-Critical Philosophy Chicago, Illinois, University of Chicago Press.

Solow,R. (1987, 12 de julio). We'd better watch out. New York Book Review (p. 36). Recuperado de http://www.standupeconomist.com/pdf/misc/solow -computer-productivity.pdf.

Tricoci, G. (2011). Las TIC y el conocimiento. Un enfoque económico y de negocios ( $2^{\mathrm{a}}$ edición). Ediciones Cooperativas, C.A.B.A. Argentina.

Van Dijk, J. y Hacker, K. (2003). The digital divide as a complex and dynamic phenomenon. The Information Society, 19. 315-326.

\section{Información del autor de correspondencia}

Guillermo A. Tricoci.

tricoci@economicas.uba.ar.

Contador Público. Licenciado en Administración de Empresas Postgrado en Economía. Sub-Director del Departamento Pedagógico de Sistemas, Facultad de Ciencias Económicas, Universidad de Buenos Aires. Profesor Titular (int) de Tecnología de la Información. Director de Proyectos de Investigación en TIC. Consultor en el sector público y privado. 